\title{
Prognostic Factors in Diffuse Large B-cell Lymphoma
}

\author{
Nozomi Niitsu
}

(DOI: 10.2169/internalmedicine.45.0142)

Diffuse large B-cell lymphoma (DLBCL) is the most common type of adult lymphoma and accounts for $40-50 \%$ of non-Hodgkin's lymphomas. Approximately half of the patients can be cured by chemotherapy. As a prognostic factor of non-Hodgkin's lymphoma, the International Prognostic Index (IPI) is generally used (1). IPI is a representative prognostic factor, which was proposed as a prognosis predicative model for aggressive lymphoma treated with a CHOP (cyclophosphamide, doxorubicin, vincristine, prednisolone) regimen or regimens similar to CHOP. Currently, the IPI is widely used in determining a treatment plan. As a result, the five prognostic factors shown in Table 1 were sampled as independent factors for the total survival rate, and the prognosis predictive model was made, in which four groups were classified based on the number of factors. The complete remission rates of each risk group by IPI from lowest risk to highest were $87 \%, 67 \%, 55 \%$, and $44 \%$, respectively. The 5-year survival rates were $73 \%, 51 \%, 43 \%$, and $26 \%$, respectively. For patients of age 60 years or younger, the three factors: stage, serum $\mathrm{LDH}$, and performance status (PS) were considered as independent prognostic factors and were called the age-adjusted international index (AAII). The 5 -year survival rates rated by AAII were $83 \%, 69 \%, 46 \%$, and $32 \%$, respectively. Therefore, in a similar manner, the low risk group had a better prognosis and the high risk group had a poor prognosis. The survival curves by prognostic factors in patients with DLBCL are shown in Fig. 1. Patients with a better prognosis and a poor prognosis are seen in the same risk group. The reason is thought to be that DLBCL is not a single disease, but a heterogeneous group, which has a variety of cell morphologies and different biological characteristics, such as gene expression patterns. Tomita et al (2) treated patients with DLBCL with a stratified protocol, sampled the serum LDH and PS as prognostic factors, and reported the same results as seen with the IPI.

$$
\text { See also p } 247 .
$$

DOI: 10.2169/internal medicine.45.1549

Recently, the prognostic factors for DLBCL have been evaluated using DNA microarrays. A DNA microarray con- sists of thousands or tens of thousands of nucleic acid probes arranged on a single plate by which molecular identification through analysis of gene expression patterns is possible using gene expression profiling. It is expected to become a very useful technique in cancer gene research. Alizadeh et al (3) identified two types of DLBCL which have different states of differentiation based on the gene expression of corresponding normal B-cells, and reported that patients with germinal center B-like (GCB) DLBCL, which showed the gene expression profiles of germinal center Bcells, had a significantly better prognosis than those with activated B-like (ABC) DLBCL, which showed gene expression profiles similar to mitogen-activated peripheral B-cells in vitro. Later, they evaluated the state of the somatic mutations in the immunoglobulin heavy chain genes in these patients and reported that DLBCL, which showed these mutations, belonged to the GCB group. All lymphomas that had $\mathrm{t}$ $(14 ; 18)(\mathrm{q} 32 ; \mathrm{q} 21)$ belonged to the GCB group and all CD10 positive cases also belonged to the GCB group. It was seen especially in the $t(14 ; 18)$ positive group. Thus, the GCB group of DLBCL was thought to be derived from germinal center B-cells and the ABC group of DLBCL was thought to be a new lymphoma group. However, this technique is very expensive and there are no practical methods

Table 1. International Prognostic Index

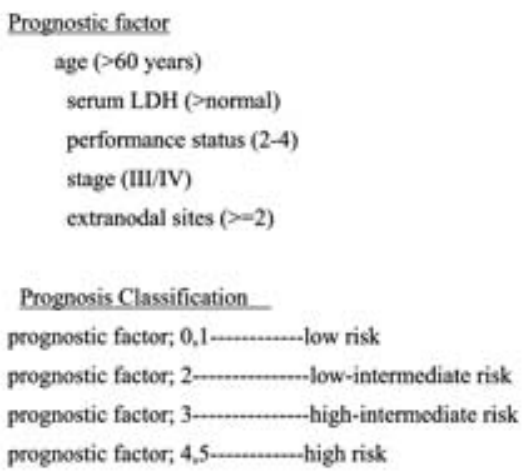



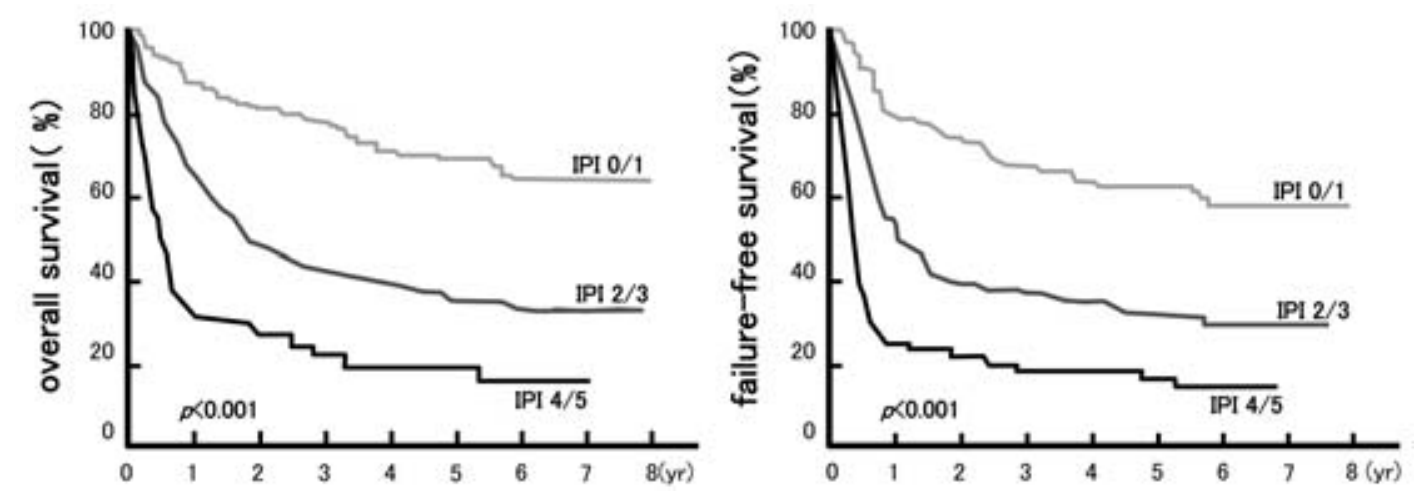

Figure 1. Overall survival of patients with DLBCL according to the international Prognostic index

suitable to genome-scale analysis. Therefore, the use of this technique is limited currently.

Lossos et al (4), using quantitative real-time PCR, evaluated 36 sets of gene expression that are reported to predict the survival period in patients with DLBCL. The results showed that the top 6 genes that predict prognosis most closely were $L M O 2, B C L 6, F N 1, C C N D 2, S C Y A 3$, and $B C L$ 2. When the expression of $L M O 2, B C L 6$, and FN1 were high, the prognosis was better, whereas when the expression of $C C N D 2, S C Y A 3$, and $B C L 2$ were high, the prognosis was poor. $L M O 2$ and $B C L 6$ show high expression in germinal center B cell-like and CCND2, SCYA3, and BCL2 show high expression in activated B cell-like. Therefore, a prognosis curve was seen similar to those which have been reported previously. In conclusion, it appears possible to predict the survival rate of DLBCL by measuring the expression of 6 genes, and it is expected that this approach will be applied clinically in the future.

\section{References}

1. Shipp MA. A predictive model for aggressive non-Hodgkin's lymphomas. The International Non-Hodgkin's Lymphoma Prognostic Factors Project. N Engl J Med 329: 987-994, 1993.

2. Tomita N, et al. Prognostic factors in diffuse large B-cell lymphoma treated by resk-adopted therapy. Intern Med 45: 247-252, 2006.
3. Alizadeh AA, Eisen MB, Davis RE, et al. Distinct type of diffuse large B-cell lymphoma identified by gene expression profiling. Nature 403: 503-511, 2000.

4. Lossos IS, Czerwinski DK, Alizadeh AA, et al. Prediction of survival in diffuse large-B-cell lymphoma based on the expression of six genes. N Engl J Med 350: 1828-1837, 2004.

(C) 2006 The Japanese Society of Internal Medicine http://www.naika.or.jp/imindex.html 